\title{
QCD DIRAC SPECTRA AND THE TODA LATTICE*
}

\author{
K. SPLITTORFF \\ Nordita, Blegdamsvej 17, \\ DK-2100, Copenhagen, Denmark \\ split@alf.nbi.dk \\ J. J. M. VERBAARSCHOT \\ Department of Physics and Astronomy, \\ SUNY at Stony Brook, Stony Brook, NY11794, US \\ jacobus.verbaarschot@stonybrook.edu
}

\begin{abstract}
We discuss the spectrum of the QCD Dirac operator both at zero and at nonzero baryon chemical potential. We show that, in the ergodic domain of QCD, the Dirac spectrum can be obtained from the replica limit of a Toda lattice equation. At zero chemical potential this method explains the factorization of known results into compact and noncompact integrals, and at nonzero chemical potential it allows us to derive the previously unknown microscopic spectral density.
\end{abstract}

\section{Introduction}

Because of the spontaneous breaking of chiral symmetry and confinement, QCD at low energy is a theory of weakly interacting Goldstone bosons. In the spontaneously broken phase, the QCD partition function is a nonanalytic function of the quark mass with a chiral condensate that is discontinuous as the quark mass crosses the eigenvalue axis of the QCD Dirac operator. The strength of this discontinuity is proportional to the eigenvalue density, a relation known as the Banks-Casher formula ${ }^{1}$.

A theory with spontaneously broken chiral symmetry that is much simpler than QCD is chiral Random Matrix Theory ${ }^{2}$. This is a theory with the global flavor symmetries of QCD in which the matrix elements of the Dirac operator are replaced by random numbers. Although, this theory is zero dimensional, chiral symmetry is broken spontaneously in the limit

*This work is supported in part by US DOE grant No. DE-FG-88ER40388. 
of infinitely large matrices, and the mass of the non-Goldstone modes diverges in the limit $N \rightarrow \infty$, where $N$ is the matrix size. Therefore, in the thermodynamic limit, $N \rightarrow \infty$, chiral Random Matrix theory reduces to a theory of Goldstone bosons for which, in the limit of small quark masses, the Lagrangian is just the mass term of the chiral Lagrangian. This is the main reason why Random Matrix Theories have been so successful in this context.

One of the questions we have been asking is whether we can identify a parameter domain where QCD and chiral Random Matrix Theory reduce to the same theory of Goldstone bosons. The affirmative answer to this question is that this is the case if the Compton wavelength of the Goldstone bosons is much larger than the linear size $L$ of the box. This requires that the quark masses $m_{f} \ll F^{2} /\left(\Sigma L^{2}\right)$, which is an unphysical domain of QCD, so that the kinetic term of the chiral Lagrangian can be ignored ${ }^{3,4}$. ( $\Sigma$ is the chiral condesate and $F$ is the pion decay constant.) However, even for realistic quark masses we can identify a parameter domain where QCD and chiral Random Matrix Theory behave the same, namely the domain where eigenvalues of the Dirac operator $\lambda \ll F^{2} /\left(\Sigma L^{2}\right)$ which is known as the ergodic domain ${ }^{5}$. The reason is that the generating function of the Dirac spectrum is a QCD-like partition function with additional ghost quarks with mass $\lambda$. The condition for the validity of the Random Matrix Theory description of the Dirac spectrum is then that the Compton wavelength of Goldstone bosons composed out of ghost quarks is much larger than the size of the box. Indeed, such behavior has been observed in numerous lattice QCD simulations (as discussed in detail elsewhere ${ }^{6,7}$ ).

At nonzero chemical potential the eigenvalues of the Dirac operator are scattered in the complex plane. It has been shown that the Dirac spectrum remains in the ergodic domain if the inverse chemical potential is much larger than the size of the box ${ }^{8}$. In this domain the Dirac spectrum can be described by a chiral Random Matrix Model that has been extended with a chemical potential ${ }^{9,10}$. However, until recently, this random matrix model had only been solved at the mean field level ${ }^{9}$. The standard methods to derive nonperturbative results such as the supersymmetric method ${ }^{11}$ and complex orthogonal polynomial methods ${ }^{12,13,14}$ have not been successful in this case. So far the supersymmetric method failed because of technical problems in calculating the graded integrals, while the method of complex orthogonal polynomials failed because of the absence of an eigenvalue representation at non-zero chemical potential. The mean field analysis of the random matrix model was performed using the replica trick ${ }^{15}$ which was 
widely believed to only work for the derivation of perturbative results ${ }^{16}$. However, it was shown recently that if a family of partition functions has certain integrability properties it is possible to obtain exact nonperturbative results by means of the replica trick ${ }^{17,18}$. We will show that the ergodic limit of the phase quenched QCD partition function at nonzero chemical potential has the required integrability properties ${ }^{19}$ so that the hierarchy of phase quenched partition functions with a different number of flavors are related by a recursion relation ${ }^{19}$ which is known as the Toda lattice equation. The spectral density is then obtained from the replica limit of this Toda lattice equation.

In the first part of this lecture we discuss the Dirac spectrum at zero chemical potential. We show that in the ergodic domain the spectral density can be obtained from the replica limit of the Toda lattice equation. This result explains a factorization property of the resolvent. In the second half of this lecture we study the quenched Dirac spectrum at nonzero chemical potential. Using the replica limit of the Toda lattice equation we derive the analytical result for the microscopic spectral density in the ergodic domain.

\section{The Dirac Spectrum in QCD}

The eigenvalues $\left\{\lambda_{k}\right\}$ of the anti-hermitian Dirac operator are determined by the eigenvalue equation

$$
D \phi_{k}=i \lambda_{k} \phi_{k} .
$$

Because of the axial symmetry the nonzero eigenvalues occur in pairs $\pm \lambda_{k}$. The number of zero eigenvalues is almost always equal to the topological charge of the gauge field configuration. The average spectral density, defined by

$$
\rho(\lambda)=\left\langle\sum_{k} \delta\left(\lambda-\lambda_{k}\right)\right\rangle,
$$

can be obtained from the discontinuity of the average resolvent

$$
G(z)=\left\langle\sum_{k} \frac{1}{z+i \lambda_{k}}\right\rangle,
$$

where, in both cases, the average is over gauge field configurations distributed according to the QCD action. This can be seen by considering the rectangular contour in Fig. 1. Assuming that the average spectral density does not vary substantially along this contour, the average total number of 

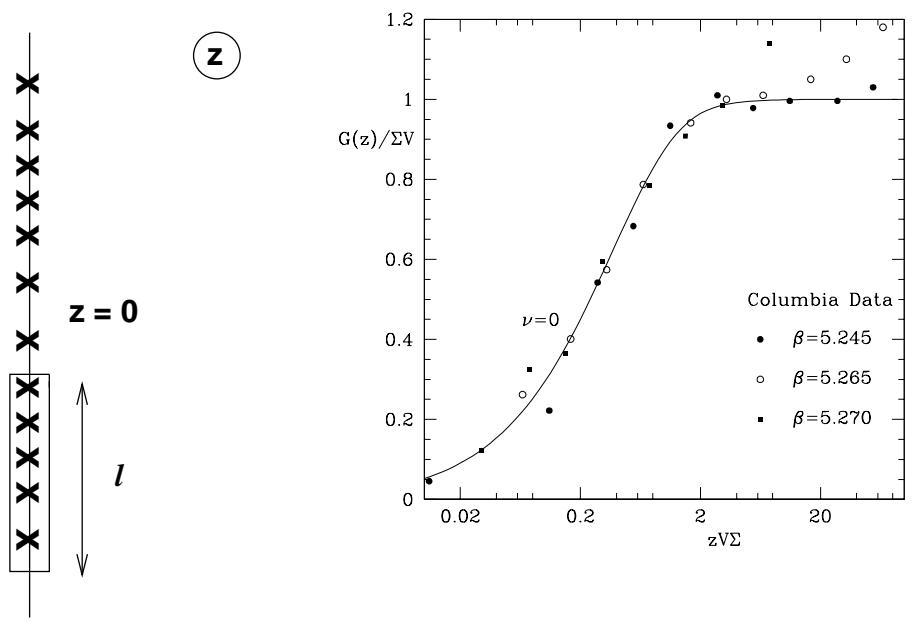

Figure 1. A typical Dirac spectrum (left) and the average resolvent $G(z)$ in units of $\Sigma V$ compared with lattice QCD data.

eigenvalues inside this contour is $\rho(\lambda) l$, where $\lambda$ is a point on the imaginary axis inside this contour. Therefore, if we integrate the resolvent along this contour, we obtain

$$
\oint G(z)=i l(G(i \lambda+\epsilon)-G(i \lambda-\epsilon))=2 \pi i \rho(\lambda) l
$$

Using the symmetry of the spectrum we find ${ }^{1}$

$$
\operatorname{Re}[G(i \lambda+\epsilon)]=\pi \rho(\lambda) .
$$

In QCD, the chiral condensate defined by $\Sigma=G(\epsilon) / V$ where $V$ is the volume of space time, is nonzero because of the spontaneous breaking of chiral symmetry. The average level spacing near zero is therefore given by

$$
\Delta=\frac{1}{\rho(0)}=\frac{\pi}{\Sigma V}
$$

which is also the scale of the smallest nonzero eigenvalue. Asymptotically, for large $\lambda$, the Dirac eigenvalues approach the spectrum of a free Dirac operator with eigenvalue density given by $\sim V \lambda^{3}$. 
Because the eigenvalues near zero are spaced as $1 / \Sigma V$ it makes sense to introduce the microscopic spectral density ${ }^{2}$

$$
\rho_{s}(\lambda)=\lim _{V \rightarrow \infty} \frac{1}{V \Sigma} \rho\left(\frac{\lambda}{V \Sigma}\right) .
$$

In the ergodic domain of QCD this is a universal function that can also be derived from chiral Random Matrix Theory.

\subsection{Ergodic Domain of $Q C D$}

The low-energy limit of QCD for $N_{f}$ fermionic flavors is described by a theory of weakly interacting Goldstone bosons parametrized by the unitary matrix $U\left(N_{f}\right)$. If their mass

$$
m_{\pi}^{2}=\frac{2 m \Sigma}{F^{2}} \ll \frac{\pi^{2}}{L^{2}}
$$

(with $F$ the pion decay constant) the kinetic term of the chiral Lagrangian factorizes from the partition function and the mass dependence of the partition function in the sector of topological charge $\nu$ is given by ${ }^{20}$

$$
Z_{N_{f}}^{\nu}(M)=\int_{U\left(N_{f}\right)} \operatorname{det}^{\nu} U e^{\frac{1}{2} \Sigma V \operatorname{Tr}\left[M U^{-1}+M^{\dagger} U\right]},
$$

where the quark mass matrix is defined by $M=\operatorname{diag}\left(m_{1}, \cdots, m_{N_{f}}\right)$. This is the ergodic domain of QCD.

As we will explain below partition functions with bosonic quarks are essential for obtaining the resolvent and average spectral density of the Dirac operator. For bosonic quarks, the Goldstone bosons cannot be parameterized by a unitary matrix. The reason is that symmetry transformations have to be consistent with the convergence of the bosonic integrals. Let us consider the case of one bosonic flavor. Then

$$
\operatorname{det}^{-1}\left(\begin{array}{cc}
m & i d \\
i d^{\dagger} & m
\end{array}\right)=\frac{1}{\pi^{2}} \int d^{2} \phi_{1} d^{2} \phi_{2} \exp \left[-\left(\begin{array}{c}
\phi_{1}^{*} \\
\phi_{2}^{*}
\end{array}\right)^{T}\left(\begin{array}{cc}
m & i d \\
i d^{\dagger} & m
\end{array}\right)\left(\begin{array}{c}
\phi_{1} \\
\phi_{2}
\end{array}\right)\right]
$$

so that the exponent is purely imaginary for $m=0$ and convergent for $\operatorname{Re}(m)>0$.

The most general flavor transformation of the action in (10) is a $G l(2)$ transformation that can be parameterized as

$$
U=e^{H} V \quad \text { with } \quad H^{\dagger}=H \quad \text { and } \quad V V^{\dagger}=1 .
$$


For $U$ to be a symmetry transformation for $m=0$ we require that

$$
U^{\dagger}\left(\begin{array}{cc}
0 & i d \\
i d^{\dagger} & 0
\end{array}\right) U=\left(\begin{array}{cc}
0 & i d \\
i d^{\dagger} & 0
\end{array}\right)
$$

so that $H$ has to be a multiple of $\sigma_{3}$ and $V$ has to be a multiple of the identity. The $V$ part of $U$ is not broken by the mass term and is thus a vector symmetry. Only the symmetry transformation $\exp \left(s \sigma_{3}\right)$ is broken by the mass term so that the axial transformations can be parameterized by

$$
U=\left(\begin{array}{cc}
e^{s} & 0 \\
0 & e^{-s}
\end{array}\right) \quad \text { with } \quad s \in\langle-\infty, \infty\rangle .
$$

For $N_{f}$ bosonic flavors the axial transformations are parameterized by

$$
U=\left(\begin{array}{cc}
e^{H} & 0 \\
0 & e^{-H}
\end{array}\right) \quad \text { with } \quad H^{\dagger}=H .
$$

The Goldstone manifold is thus $G l\left(N_{f}\right) / U\left(N_{f}\right)$. In the domain were the kinetic term of the chiral Lagrangian can be ignored, the effective bosonic partition function (indicated by the subscript $-N_{f}$ ) in the sector of topological charge $\nu$ is given by ${ }^{21,22,23}$

$$
Z_{-N_{f}}^{\nu}\left(m_{f}\right)=\int_{G l\left(N_{f}\right) / U\left(N_{f}\right)} \operatorname{det}^{\nu} U e^{\frac{1}{2} \Sigma V \operatorname{Tr}\left[M U^{-1}+M^{\dagger} U\right]} .
$$

The resolvent can be obtained from a supersymmetric generating function that contains one additional fermionic ghost quark and one additional bosonic ghost quark,

$$
G(z)=\left.\partial_{z}\left\langle\frac{\operatorname{det}(D+z)}{\operatorname{det}\left(D+z^{\prime}\right)}\right\rangle\right|_{z^{\prime}=z} .
$$

Therefore the low energy limit of this generating function contains additional ghost Goldstone bosons and fermions with mass given by $2 z \Sigma / F^{2}$. For $z \ll F^{2} / \Sigma L^{2}$ the $z$-dependence of this generating function is given by 21,22

$$
Z_{N_{f}+1 ;-1}^{\nu}(M)=\int_{\hat{G} l\left(N_{f}+1 \mid 1\right)} \operatorname{det}^{\nu} U e^{\frac{1}{2} \Sigma V \operatorname{STr}\left[M U^{-1}+M^{\dagger} U\right]},
$$

with $M=\operatorname{diag}\left(m_{1}, \cdots, m_{N_{f}}, z, z^{\prime}\right)$ and $\hat{G} l\left(N_{f}+1 \mid 1\right)$ are super-matrices with a unitary $U\left(N_{f}+1\right)$ upper left block, a $G l(1) / U(1)$ lower right block and Grassmann valued matrix elements elsewhere. The number of QCD Dirac eigenvalues that is described by this partition function is of the order

$$
\frac{F^{2}}{\Sigma L^{2} \Delta}=F^{2} L^{2}
$$


This number increases linearly in $N_{c}$ for $N_{c} \rightarrow \infty$ which was recently confirmed by lattice simulations ${ }^{24}$.

An alternative to the supersymmetric method is to use the replica trick to calculate the resolvent. It comes in two different versions: the fermionic replica trick defined by

$$
G(z)=\lim _{N_{f} \rightarrow 0} \frac{1}{N_{f}} \log Z_{N_{f}}^{\nu}(z),
$$

and the bosonic replica trick defined by

$$
G(z)=\lim _{N_{f} \rightarrow 0} \frac{1}{-N_{f}} \log Z_{-N_{f}}^{\nu}(z) .
$$

If we take the replica limit of the fermionic (19) or bosonic (20) partition functions directly, we will obtain a result that differs from the supersymmetric calculation ${ }^{16}$. For almost two decades there were no methods to do reliable nonperturbative calculations with the replica trick. In the next section we will show that these problems with the replica trick can be avoided if the take the replica limit of the Toda lattice equation.

\subsection{Toda Lattice Equation}

We now consider bosonic and fermionic partition functions with all masses equal to $z$ which only depend on the combination, cf. (9) and (15),

$$
x=z \Sigma V .
$$

The unitary integral in the fermionic partition function (9) can be evaluated by decomposing $U=V \operatorname{diag}\left(e^{i \theta_{k}}\right) V^{\dagger}$ and choosing the $\left\{\theta_{k}\right\}$ and $V$ as new integration variables. By expanding the Jacobian of this transformation, given by $\prod_{k<l}\left|\exp \left(i \theta_{k}\right)-\exp \left(i \theta_{l}\right)\right|^{2}$, the different terms factorize into products of modified Bessel functions which can be combined again into a single determinant. The final result is given by 25,26

$$
Z_{N_{f}}^{\nu}(x)=\operatorname{det}\left[I_{\nu+k-l}(x)\right]_{k, l=1, \cdots, N_{f}} .
$$

By using recursion relations for the Bessel functions, this result can be rewritten as

$$
Z_{N_{f}}^{\nu}(x)=\frac{1}{x^{N_{f}\left(N_{f}-1\right)}} \operatorname{det}\left[\left(x \partial_{x}\right)^{k+l} I_{\nu}(x)\right]_{k, l=0, \cdots, N_{f}-1} .
$$

Next we use the Sylvester identity ${ }^{27,28}$ which is valid for determinant of an arbitrary matrix $A$. It is given by

$$
C_{i j} C_{p q}-C_{i q} C_{p j}=\operatorname{det}(A) C_{i j ; p q},
$$


where the $C_{i j}$ are cofactors of the matrix $A$ and the $C_{i j ; p q}$ are double cofactors. By applying this identity to the determinant in (23) for $i=j=$ $N_{f}-1$ and $p=q=N_{f}$, we easily derive the Toda lattice equation ${ }^{29,30,31}$

$$
\left(x \partial_{x}\right)^{2} \log Z_{N_{f}}^{\nu}(x)=2 N_{f} x^{2} \frac{Z_{N_{f}+1}^{\nu}(x) Z_{N_{f}-1}^{\nu}(x)}{\left[Z_{N_{f}}^{\nu}(x)\right]^{2}} .
$$

This equation has also been derived as a consistency condition for QCD partition functions ${ }^{32}$. It also occurs in other application such as for example in self-dual Chern-Simons theory ${ }^{33}$.

In the case of bosonic quarks (15) the positive definite matrix is diagonalized as $U=V \operatorname{diag}\left(e^{s_{k}}\right) V^{\dagger}$. Choosing the $\left\{s_{k}\right\}$ and $V$ as new integration variables, we obtain ${ }^{23}$ after expanding the Jacobian given by $\prod_{k<l}\left(\exp \left(s_{k}\right)-\exp \left(s_{l}\right)\right)\left(\exp \left(-s_{k}\right)-\exp \left(-s_{l}\right)\right)$,

$$
Z_{-N_{f}}^{\nu}(x)=\operatorname{det}\left[K_{\nu+k-l}(x)\right]_{k, l=1, \cdots, N_{f}} \text {. }
$$

As in the fermionic case, this result can be written in the form of a $\tau$ function

$$
Z_{-N_{f}}^{\nu}(x)=\frac{1}{x^{N_{f}\left(N_{f}-1\right)}} \operatorname{det}\left[\left(x \partial_{x}\right)^{k+l} K_{\nu}(x)\right]_{k, l=0, \cdots, N_{f}-1},
$$

where we have used that $I_{\nu}$ and $(-1)^{\nu} K_{\nu}$ satisfy the same recursion relations. Therefore, the bosonic partition function also satisfies the Toda lattice equation (25).

The two semi-infinite hierarchies are connected by

$$
\lim _{N_{f} \rightarrow 0} \frac{1}{N_{f}}\left(x \partial_{x}\right)^{2} \log Z_{N_{f}}^{\nu}(x) .
$$

By extending the Toda lattice hierarchy to include an additional spectator boson, it can be shown that ${ }^{34}$

$$
\begin{aligned}
\lim _{N_{f} \rightarrow 0} \frac{1}{N_{f}}\left(x \partial_{x}\right)^{2} \log Z_{N_{f}}^{\nu}(x) & =\lim _{y \rightarrow x} x \partial_{x}\left(x \partial_{x}+y \partial_{y}\right) \log Z_{1,-1}^{\nu}(x \mid y) \\
& =x \partial_{x} \lim _{y \rightarrow x} x \partial_{x} \log Z_{1,-1}^{\nu}(x \mid y) \\
& =x \partial_{x} x G(x) .
\end{aligned}
$$

Taking the replica limit of the Toda lattice equation (25) we thus obtain the identity

$$
x \partial_{x} x G(x)=2 x^{2} Z_{1}^{\nu}(x) Z_{-1}^{\nu}(x),
$$

which explains this factorization property. In the same way we can show the factorization of the susceptibility into a bosonic and a fermionic partition function ${ }^{19}$. 
Inserting the expressions for $Z_{1}$ and $Z_{-1}$ we find

$$
G(x)=x\left(K_{\nu}(x) I_{\nu}(x)+K_{\nu-1}(x) I_{\nu+1}(x)\right)+\frac{\nu}{x} .
$$

For $\nu=0$ this result is shown as $G(x) / \Sigma V$ by the solid curve in the right figure of Fig. 1. Agreement with lattice data ${ }^{35}$ is found in the ergodic domain of QCD.

\section{Dirac Spectrum at Nonzero Chemical Potential}

At nonzero baryon chemical potential the Dirac operator is modified according to

$$
D \rightarrow D+\mu \gamma_{0}
$$

This Dirac operator does not have any hermiticity properties and its eigenvalues are scattered in the complex plane $36,37,38,39,40$. For small $\mu$ we expect that the width of the cloud of eigenvalues ${ }^{36} \sim \mu^{2}$. The average spectral density is given by

$$
\rho(\lambda)=\left\langle\sum_{k} \delta^{2}\left(\lambda-\lambda_{k}\right)\right\rangle,
$$

and the average resolvent is defined as usual by (3). They are related by

$$
\left.\partial_{z^{*}} G(z)\right|_{z=\lambda}=\pi \rho(\lambda) .
$$

The quenched spectral density is therefore given by the replica limit $41,42,9$

$$
\rho\left(z, z^{*}\right)=\lim _{n \rightarrow 0} \frac{1}{\pi n} \partial_{z} \partial_{z^{*}} \log Z_{n}\left(z, z^{*}\right)
$$

with generating function given by (note that $n$ counts pairs of quarks)

$$
Z_{n}\left(z, z^{*}\right)=\left\langle\operatorname{det}^{n}\left(D+\mu \gamma_{0}+z\right) \operatorname{det}^{n}\left(-D+\mu \gamma_{0}+z^{*}\right)\right\rangle .
$$

The low-energy limit of this generating function is a chiral Lagrangian which is determined by its global symmetries and transformation properties. By writing the product of the two determinants as ${ }^{8}$

$$
\operatorname{det}\left(D+\mu \gamma_{0}+z\right) \operatorname{det}\left(-D+\mu \gamma_{0}+z^{*}\right)=\left|\begin{array}{cccc}
i d+\mu & 0 & z & 0 \\
0 & i d-\mu & 0 & z^{*} \\
z & 0 & i d^{\dagger}+\mu & 0 \\
0 & z^{*} & 0 & i d^{\dagger}-\mu
\end{array}\right|
$$


we observe that the $U(2) \times U(2)$ flavor symmetry is broken by the chemical potential term and the mass term. Invariance is recovered by transforming the mass term as in the case of zero chemical potential and the chemical potential term by a local gauge transformation. In the domain of $\mu$ and $z$ where we can neglect the kinetic terms, the partition function is given by 8, ?

$$
Z_{n}\left(z, z^{*}\right)=\int_{U(2 n)} d U e^{-\frac{F^{2} \mu^{2} V}{4} \operatorname{Tr}[U, B]\left[U^{-1}, B\right]+\frac{\Sigma V}{2} \operatorname{Tr} M\left(U+U^{-1}\right)},
$$

where

$$
B=\left(\begin{array}{cc}
\mathbf{1}_{n} & 0 \\
0 & -\mathbf{1}_{n}
\end{array}\right), \quad M=\left(\begin{array}{cc}
z \mathbf{1}_{n} & 0 \\
0 & z^{*} \mathbf{1}_{n}
\end{array}\right)
$$

\subsection{Integration Formula}

We have proved the following integration formula ${ }^{19}$

$$
\begin{aligned}
& \int_{U(2 n)} d U \operatorname{det}^{\nu} U e^{\frac{1}{2} \operatorname{Tr}\left[M\left(U+U^{-1}\right)\right]+\sum_{p} a_{p} \operatorname{Tr}\left[\left(U B U^{-1} B\right)^{p}\right]} \\
= & \frac{c_{n}}{(x y)^{n(n-1)}} \operatorname{det}\left[\left(x \partial_{x}\right)^{k}\left(y \partial_{y}\right)^{l} Z_{1}^{\nu}(x, y)\right]_{0 \leq k, l \leq n-1},
\end{aligned}
$$

where

$$
Z_{1}^{\nu}(x, y)=\int_{0}^{1} \lambda d \lambda I_{\nu}(\lambda x) I_{\nu}(-\lambda y) e^{2 \sum_{p} a_{p} \cos \left(2 p \cos ^{-1} \lambda\right)},
$$

and $c_{n}$ is an $n$-dependent constant. For example, consider the case $n=1$ and all $a_{p}=0$. Then the integral is given by $Z_{1}(x, y)$ which is a known integral given by

$$
\begin{aligned}
\left.Z_{1}^{\nu}(x, y)\right|_{a_{p}=0} & =\int_{0}^{1} \lambda d \lambda I_{\nu}(\lambda x) I_{\nu}(-\lambda y) \\
& =\frac{y I_{\nu}(x) I_{\nu-1}(-y)+x I_{\nu-1}(x) I_{\nu}(-y)}{x^{2}-y^{2}},
\end{aligned}
$$

which is a known result for the QCD partition function with two different masses ${ }^{44}$.

\subsection{Toda Lattice Equation}

Using the integration formula of previous section we find that the lowenergy limit of the phase quenched QCD partition function at nonzero chemical potential is given by

$$
Z_{n}^{\nu}\left(z, z^{*}\right)=\frac{c_{n}}{\left(z z^{*}\right)^{n(n-1)}} \operatorname{det}\left[\left(z \partial_{z}\right)^{k}\left(z^{*} \partial_{z^{*}}\right)^{l} Z_{1}^{\nu}\left(z, z^{*}\right)\right]_{0 \leq k, l \leq n-1},
$$


where

$$
Z_{1}^{\nu}\left(z, z^{*}\right)=\int_{0}^{1} \lambda d \lambda e^{-2 V F^{2} \mu^{2}\left(\lambda^{2}-1\right)}\left|I_{\nu}(\lambda z V \Sigma)\right|^{2} .
$$

Using the Sylvester identity as at zero chemical potential we obtain the Toda lattice equation

$$
z \partial_{z} z^{*} \partial_{z^{*}} \log Z_{n}^{\nu}\left(z, z^{*}\right)=\frac{\pi n}{2}\left(z z^{*}\right)^{2} \frac{Z_{n+1}^{\nu}\left(z, z^{*}\right) Z_{n-1}^{\nu}\left(z, z^{*}\right)}{\left[Z_{n}^{\nu}\left(z, z^{*}\right)\right]^{2}} .
$$

The spectral density (35) follows from the replica limit of this equation. Using $Z_{0}^{\nu}\left(z, z^{*}\right)=1$ we find the simple expression

$$
\rho\left(z, z^{*}\right)=\lim _{n \rightarrow 0} \frac{1}{\pi n} \partial_{z} \partial_{z^{*}} \log Z_{n}^{\nu}\left(z, z^{*}\right)=\frac{z z^{*}}{2} Z_{1}^{\nu}\left(z, z^{*}\right) Z_{-1}^{\nu}\left(z, z^{*}\right) .
$$

What remains to be done is to calculate the partition function with one bosonic and one conjugate bosonic quark which will be completed in the next subsection.

\subsection{The Bosonic Partition Function}

In this subsection we evaluate the low-energy limit of the QCD partition function at nonzero chemical potential for one bosonic quark and one conjugate bosonic quark. Because of convergence requirements it is more complicated to derive the chiral Lagrangian in this case. By a careful analysis we find ${ }^{19}$,

$$
Z_{-1}^{\nu}\left(z, z^{*}\right)=\int_{U \in G l(2) / U(2)} d U \operatorname{det}^{\nu} U e^{-\frac{F^{2} \mu^{2} V}{4} \operatorname{Tr}[U, B]\left[U^{-1}, B\right]+\frac{i \Sigma V}{2} \operatorname{Tr} \zeta^{T}\left(U-I U^{-1} I\right)},
$$

where $B$ is the baryon number matrix defined in (39) and the mass matrix $\zeta$ and the anti-symmetric matrix $I$ are defined as

$$
\zeta=\left(\begin{array}{cc}
\epsilon & z \\
z^{*} & \epsilon
\end{array}\right) \quad \text { and } \quad I=\left(\begin{array}{cc}
0 & 1 \\
-1 & 0
\end{array}\right) .
$$

Although this integral is convergent for $\operatorname{Im} \epsilon>0$, it diverges logarithmically for $\epsilon \rightarrow 0$. We have also ${ }^{19}$ derived the partition function (47) starting from a chiral Random Matrix Theory at nonzero chemical potential and using the Ingham-Siegel integral ${ }^{45}$.

The integral (47) can be evaluated analytically by using an explicit parameterization of positive definite $2 \times 2$ matrices. We find

$$
Z_{-1}^{\nu}(z=x+i y)=C_{-1} \log \epsilon e^{\frac{V \Sigma^{2}\left(y^{2}-x^{2}\right)}{4 \mu^{2} F^{2}}} K_{\nu}\left(\frac{V \Sigma^{2}\left(x^{2}+y^{2}\right)}{4 \mu^{2} F^{2}}\right) .
$$


The final result for the quenched spectral density is given by 19

$$
\begin{aligned}
\rho(x, y)= & \frac{V^{3} \Sigma^{4}}{2 \pi F^{2} \mu^{2}}\left(x^{2}+y^{2}\right) e^{\frac{V \Sigma^{2}\left(y^{2}-x^{2}\right)}{4 \mu^{2} F^{2}}} K_{\nu}\left(\frac{V \Sigma^{2}\left(x^{2}+y^{2}\right)}{4 \mu^{2} F^{2}}\right) \\
& \times \int_{0}^{1} \lambda d \lambda e^{-2 V F^{2} \mu^{2} \lambda^{2}}\left|I_{\nu}(\lambda(x+i y) V \Sigma)\right|^{2} .
\end{aligned}
$$

The constant has been chosen such that the $\mu \rightarrow 0$ limit of $\rho(x, y)$ for large $y$ is given by $\Sigma V / \pi$ (see below). The solid curve in Fig. 2 shows a graph of this result for $y=0$ and $\mu^{2} F^{2} V=16$ in terms of the ratio

$$
\rho_{s}(x, y)=\frac{\rho(x, y)}{\Sigma^{2} V^{2}}
$$

versus $x \Sigma V$ at $y=0$. The dotted curve shows the result which is obtained when the Bessel function $K_{\nu}$ is replaced by its asymptotic expansion. This result that was obtained from a nonhermitian eigenvalue model ${ }^{14}$ that is not in the universality class of QCD. An important difference between the two results is that the spectral density (50) for $y=0$ is quadratic in $x$ for $x \rightarrow 0$, whereas the result given by the dotted curve is linear in $x$ for $x \rightarrow 0$.

Taking the thermodynamic limit at fixed $z$ and $\mu$ the Bessel functions can be approximated by their asymptotic limit. This results in

$$
\rho(x, y)=\frac{V \Sigma^{2}}{4 \pi \mu^{2} F^{2}} \quad \text { for } \quad|x|<\frac{2 F^{2} \mu^{2}}{\Sigma},
$$

and $\rho(x, y)=0$ outside this strip in agreement with a mean field analysis 9,8 . For the integrated eigenvalue density we then find

$$
\int_{-\infty}^{\infty} d x \rho(x, y)=\frac{\Sigma V}{\pi}
$$

in agreement with the eigenvalue density at $\mu=0$.

\section{Conclusions}

In this work we have analyzed the ergodic domain of the QCD partition function where the pion Compton wavelength is much larger than the size of the box. In this domain the QCD partition function reduces to a theory of weakly interacting Goldstone bosons for which the kinetic term in the chiral Lagrangian can be ignored. Independent of the quark masses, the generating function for the Dirac spectrum is in this domain for sufficiently small eigenvalues.

We have shown that fermionic partition functions, bosonic partition functions and the supersymmetric partition function are connected by a 

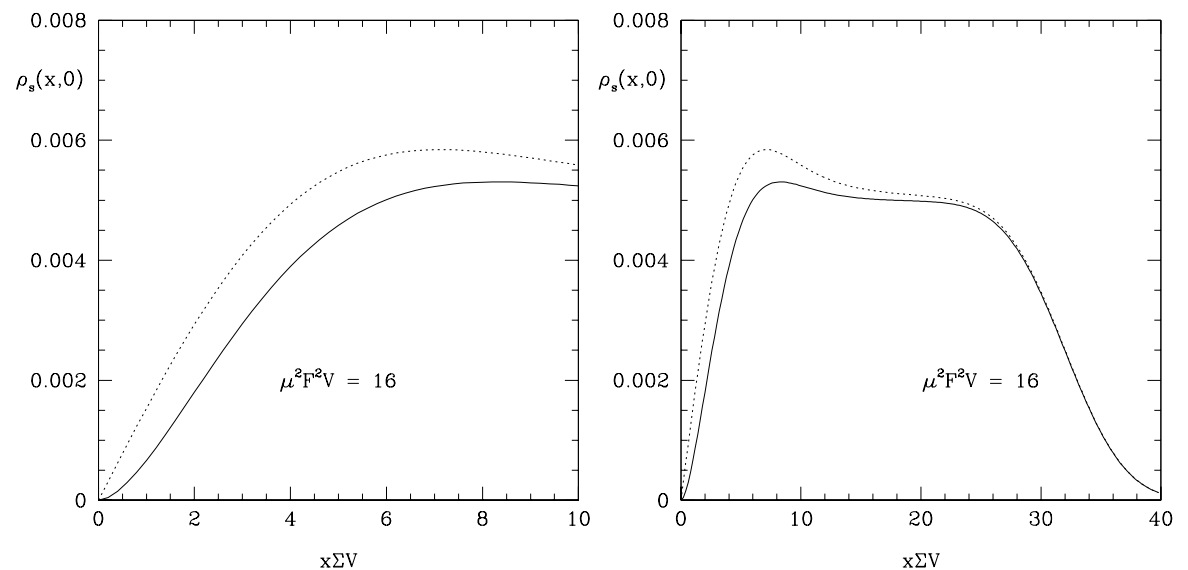

Figure 2. The quenched spectral density at nonzero chemical potential in the ergodic domain of QCD (full curve). Also shown is a result derived from an eigenvalue model (dotted curve). The left hand plot is a zoom in of the right hand one.

Toda lattice equation. This recursion relation makes it possible to derive nonperturbative results using the replica trick. In particular, this reveals the factorization of the resolvent and the susceptibility into products of simple bosonic and fermionic partition functions. In this article, we have considered the resolvent at zero chemical potential and the spectral density at nonzero chemical potential.

The resolvent for quenched QCD at nonzero chemical potential approaches zero linearly as a function of $\operatorname{Re}(z)$. In the unquenched theory, where the argument of the resolvent is also the mass in the fermion determinant, we expect a discontinuity in the resolvent as $\operatorname{Re}(z)$ crosses the imaginary axis. Can we understand the differences between these two theories in terms of the spectrum of the Dirac operator? As is suggested by earlier Random Matrix Theory simulations ${ }^{46}$ there are significant differences between the two. For example, in the unquenched theory, because of the phase of the fermion determinant, there is no reason that the spectral density is positive definite or even real. The first analytical results for the unquenched spectral density where recently obtained by James Osborn ${ }^{47}$ for a nonhermitian Random Matrix Model that is in the universality class of QCD at nonzero chemical potential. In the thermodynamic limit his results show strong oscillations but the connection with broken chiral symmetry is still a mystery. We hope to address this issue in a future publication ${ }^{48}$. 
The replica limit of the Toda lattice equation is a powerful method that is also applicable to other partition functions with an integrable structure. For example, we mention the Ginibre ensemble ${ }^{49}$, parametric correlations and the two-point function of the Gaussian Unitary Ensemble. Our experience tells us that all universal results that can be derived from a complex random matrix theory $(\beta=2)$ can also be obtained from the replica limit of a Toda lattice equation.

\section{Acknowledgments}

We thank Gernot Akemann, Gerald Dunne and James Osborn for useful discussions.

\section{References}

1. T. Banks and A. Casher, Nucl. Phys. B169 (1980) 103.

2. E.V. Shuryak and J.J.M. Verbaarschot, Nucl. Phys. A 560, 306 (1993); J.J.M. Verbaarschot, Phys. Rev. Lett. 72, 2531 (1994).

3. J. Gasser and H. Leutwyler, Ann. Phys. 158, 142 (1984); Nucl. Phys. B 250, 465 (1985); H. Leutwyler, Ann. Phys. 235, 165 (1994).

4. H. Leutwyler and A. Smilga, Phys. Rev. D 46, 5607 (1992).

5. J.J.M. Verbaarschot, Phys. Lett. B 368 (1996) 137.

6. J. J. M. Verbaarschot and T. Wettig, Ann. Rev. Nucl. Part. Sci. 50, 343 (2000).

7. P. H. Damgaard, Nucl. Phys. Proc. Suppl. 106, 29 (2002).

8. D. Toublan and J.J.M. Verbaarschot, Int. J. Mod. Phys. B 15, 1404 (2001).

9. M.A. Stephanov, Phys. Rev. Lett. 76, 4472 (1996).

10. M. A. Halasz, J. C. Osborn and J. J. M. Verbaarschot, Phys. Rev. D 56, 7059 (1997).

11. K.B. Efetov, Phys. Rev. Lett. 79, 491 (1997); Adv. Phys. 32, 53 (1983), Supersymmetry in disorder and chaos, (Cambridge University Press, Cambridge, 1997).

12. F. Di Francesco, M. Gaudin, C. Itzykson and F. Lesage, Int. J. Mod. Phys. A 94257 (1994).

13. Y.V. Fyodorov and H.-J. Sommers, J. Phys. A 36, 3303 (2003).

14. G. Akemann, Phys. Rev. Lett. 89, 072002 (2002); J.Phys. A 36, 3363 (2003).

15. S.F. Edwards and P.W. Anderson, J. Phys. F5, 965 (1975).

16. J.J.M. Verbaarschot and M.R. Zirnbauer, J. Phys. A 18, 1093 (1985).

17. E. Kanzieper, Phys. Rev. Lett. 89, 250201 (2002).

18. K. Splittorff and J.J.M. Verbaarschot, Phys. Rev. Lett. 90, 041601 (2003).

19. K. Splittorff and J.J.M. Verbaarschot, Nucl.Phys. B 683, 467 (2004).

20. J. Gasser and H. Leutwyler, Phys. lett. B 188, 477 (1987).

21. J.C. Osborn, D. Toublan and J.J.M. Verbaarschot, Nucl. Phys. B 540, 317 (1999).

22. P.H. Damgaard, J.C. Osborn D. Toublan, and J.J.M. Verbaarschot, Nucl. Phys. B 547, 305 (1999). 
23. D. Dalmazi and J.J.M. Verbaarschot, Nucl. Phys. B 592, 419 (2001).

24. R. Narayanan and H. Neuberger, arXiv:hep-lat/0405025.

25. R.C. Brower, P. Rossi and C-I. Tan, Nucl. Phys. B 190, 699 (1981); R.C. Brower and M. Nauenberg, Nucl. Phys. B 180, 221 (1981).

26. J. B. Kogut, M. Snow and M. Stone, Nucl. Phys. B 200, 211 (1982).

27. J.J. Sylvester, Compt. Rend. Acad. Sc. 54, 129 (1862).

28. P. Forrester, Log-gases and Random matrices, Web Book - available at http://www. ms. unimelb. edu. au/ matpjf/matpjf.html.

29. R. Dijkgraaf, H. Verlinde and E. Verlinde, Nucl. Phys. B 348, 435 (1991).

30. S. Kharchev, A. Marshakov, A. Mironov, A. Morozov and A. Zabrodin, Nucl. Phys. B 380, 181 (1992).

31. A. Mironov, A. Morozov and G. Semenoff, Int. J. Mod. Phys. A 11, 5031 (1996).

32. G. Akemann and P. H. Damgaard, Phys. Lett. B 432, 390 (1998).

33. G. V. Dunne, R. Jackiw, S. Y. Pi and C. A. Trugenberger, Phys. Rev. D 43, 1332 (1991) [Erratum-ibid. D 45, 3012 (1992)]; G. V. Dunne, Selfdual Chern-Simons theories, Lect. Notes Phys. M36, 1 (1995).

34. K. Splittorff and J. J. M. Verbaarschot, Nucl. Phys. B 695 (2004) 84. arXiv:hep-th/0402177.

35. S. Chandrasekharan and N. Christ, Nucl. Phys. Proc. Suppl. 47 (1996) 527.

36. I.M. Barbour, N.E. Behilil, E. Dagotto, F. Karsch, A. Moreo, M. Stone, and H.W. Wyld, Nucl. Phys. B 275 (1986) 296; M.-P. Lombardo, J.B. Kogut, and D.K. Sinclair, Phys. Rev. D 54 (1996) 2303.

37. H. Markum, R. Pullirsch and T. Wettig, Phys. Rev. Lett. 83, 484 (1999).

38. E. Bittner, M. P. Lombardo, H. Markum and R. Pullirsch, Nucl. Phys. Proc. Suppl. 106, 468 (2002).

39. E. Bittner, S. Hands, H. Markum and R. Pullirsch, Prog. Theor. Phys. Suppl. 153, 295 (2004).

40. G. Akemann and T. Wettig, Phys. Rev. Lett. 92 (2004) 102002.

41. V.L. Girko, Theory of random determinants (Kluwer Academic Publishers, Dordrecht, 1990).

42. A. Goksch, Phys. Rev. D 37 (1988) 1014.

43. J. Feinberg and A. Zee, Nucl. Phys. B 504 (1997) 579; Nucl. Phys. B 501 (1997) 643.

44. A.D. Jackson, M.K. Sener and J.J.M. Verbaarschot, Phys. Lett. B 387, 355 (1996).

45. Y. V. Fyodorov, Nucl. Phys. B 621, 643 (2002).

46. M. A. Halasz, A. D. Jackson and J. J. M. Verbaarschot, Phys. Rev. D 56, 5140 (1997).

47. J. C. Osborn, arXiv:hep-th/0403131.

48. G. Akemann, J.C. Osborn, K. Splittorff and J.J.M. Verbaarschot, in preparation.

49. E. Kanzieper, cond-mat/0312006. 